\title{
Circ-BPTF Associated Pulmonary Vascular Remodeling in COPD Via Promoting Smooth Muscle Cells Proliferation by MiR-486-5p/CEMIP Axis
}

\author{
Chang-guo Wang \\ Soochow University \\ Ying-ying Liu \\ Soochow University \\ Wei-yun Zhang \\ Soochow University \\ Meng-lan Zhang \\ Soochow University \\ Yu-ji Wang \\ Soochow University \\ Jian-an Huang \\ Soochow University \\ Ran Wang \\ Anhui Medical University \\ Jung-hong Jiang \\ Soochow University \\ Da-xiong Zeng ( $\nabla$ zengdaxiong2018@126.com ) \\ Soochow University https://orcid.org/0000-0002-6476-2974
}

\section{Research}

Keywords: pulmonary vascular remodeling, COPD, circ-BPTF, miR-486-5p, CEMIP

Posted Date: November 18th, 2021

DOI: https://doi.org/10.21203/rs.3.rs-1036371/v1

License: (c) (i) This work is licensed under a Creative Commons Attribution 4.0 International License.

Read Full License 


\section{Abstract \\ Background}

As a kind of non-coding RNA, circle RNA has been proved acting critical role in multiple diseases, including pulmonary hypertension. In this study, we investigated the role of a novel circRNA circ-BPTF, miR-486-5p and the downstream targets in pulmonary vascular remodeling of chronic obstructive pulmonary disease (COPD) patients.

\section{Methods}

A circRNA microarray was firstly used for circRNAs involved in pulmonary vascular remodeling of COPD patients. Real-time PCR and western blot analysis were performed to identify circ-BPTF, miR-486-5p and cell migration-inducing protein (CEMIP) expression. CCK-8, immunofluorescence and luciferase assay were used for exploring the role of circ-BPTF in proliferation.

\section{Results}

The circRNA microarray and real-time PCR analysis showed significant higher levels of circ-BPTF and lower miR-486-5p levels in pulmonary vessels of COPD patients. Hypoxia suppressed miR-486-5p expression but promoted expression of circ-BPTF and CEMIP in human pulmonary arterial smooth muscle cells (PASMCs) in vitro. The loss-of-function and gain-of-function experiments showed that circBPTF promoted PASMCs proliferation in vitro. Moreover, luciferase assay results indicated that circ-BPTF regulated proliferation by acting as an miR-486-5p sponge. CEMIP was identified as candidate target genes of miR-486-5p by luciferase assay.

\section{Conclusion}

Summarily, our study showed that circ-BPTF was associated with pulmonary vascular remodeling of COPD via promoting smooth muscle cells proliferation by miR-486-5p regulating CEMIP pathway.

\section{Introduction}

As a serious and progressive pulmonary vascular disease, pulmonary hypertension $(\mathrm{PH})$ is a kind of complication of multiple chronic lung diseases (Nathan et al. 2019). PH is featured with elevating pulmonary arterial pressure, which was caused by pulmonary vasoconstriction and pulmonary vascular remodeling (Poch et al. 2021). Chronic obstructive pulmonary disease (COPD) is one of the most popular disorders in respiratory system, which eventually lead to pulmonary hypertension (Gredic et al. 2021). Previous reports, including our studies, have proved that pulmonary vascular remodeling occurred in early stage of COPD (Churg et al. 2008; Liu et al. 2020; Zeng et al. 2010; Wang et al. 2018). We also 
demonstrated that cyclin D1 and NR4A3 were involved in pulmonary vascular remodeling of COPD (Liu et al. 2020; Zeng et al. 2010; Zeng et al. 2013; Wang et al. 2015; Wang et al. 2018).

Although increasing studies have demonstrated the role of multiple growth factors or pathways (such as prostacyclin, nitric oxide and endothelin, etc.) in pulmonary vascular remodeling, drugs targeting these pathways have little benefit to COPD patients with PH (Bunel et al. 2019; Pichl et al. 2019). As a critical feature of pulmonary vascular remodeling, hypertrophy of pulmonary artery smooth muscle cells (PASMCs) contributed greatly to the sustained increase of pulmonary vascular resistance and pulmonary artery pressure in PH (Blanco et al. 2016; de Jesus Perez et al. 2016). Therefore, it is essential to explore the molecular mechanisms responsible for PASMCs proliferation to ameliorate pulmonary vascular remodeling.

Circle RNA (circRNA) are RNA molecules with covalently joined 3'- and 5'- ends formed by back-splicing events (Wang et al. 2019). CircRNA are widely found in mammalian cells and exert biological effects at the posttranscriptional level by sponging miRNAs at response elements or miRNA-binding sites (Bonnet et al. 2020). Recent reports indicated that circRNA might involved in PH (Guo et al. 2021; Zhang et al. 2020; Zhou et al. 2019; Ma et al. 2020; Jin et al. 2020). Circ-calm4 has been proved promoting PASMCs proliferation via sponging miR-337-3p. Hsa_circ_0016070 and mmu_circ_0000790 were also involved in $\mathrm{PH}$ via regulating PASMCs proliferation. However, the function of circRNA in pulmonary vascular remodeling of COPD has not been clearly reported.

In the current study, we aimed to identify new functional circRNAs and clarify their roles in pulmonary vascular remodeling of COPD and hPASMCs proliferation. We found and investigated a novel circRNA circ-BPTF, which showed increased expression in COPD and hPASMCs under hypoxia. Our report might uncover a new regulatory mechanism for COPD with $\mathrm{PH}$ and provide a potential target for the treatment of this disease.

\section{Methods}

\section{Lung tissues collection}

This study was approved by the research ethics committee of the First Affiliated Hospital of Soochow University, and written informed consents were obtained from all subjects. Human peripheral lung samples were collected from patients who underwent pneumonectomy for lung volume reduction or pulmonary nodules from January 2016 to December 2018 in our hospital. Lung tissue samples were obtained from normal areas as far as possible from the pulmonary nodules (at least $2 \mathrm{~cm}$ ). Peripheral lung samples were inflated with $4 \%(\mathrm{w} / \mathrm{v})$ para-formaldehyde for embedding in paraffin or stored at $-80^{\circ} \mathrm{C}$ for protein extraction procedure. Pulmonary function tests were performed before surgery in all patients. COPD was determined by the standard of pulmonary function as FEV1/FVC less than 0.7 .

\section{Pulmonary Vascular Morphometry}


Paraffin-embeded peripheral lung samples were cut into multiple 5- $\mu$ m-thick sections. Sections were deparaffinized and stained with hematoxylin and eosin for the morphometric measurements under an optical microscope as described in our previous reports. Meanwhile, sections were stained with a-smooth muscle actin using immunohistochemistry for evaluating the muscularization ratio. The assessment was limited to medium and small arteries $(\leq 500 \mu \mathrm{m}$ diameter) with complete circumferential smooth muscle layer. At least five vessels were measured in each section.

\section{Circrna Microarray Scan}

The explants of pulmonary artery from lung samples from COPD group and non-COPD group (three samples both groups) were collected and total RNA was isolated using TRIzol reagent according to the manufacturer's protocol. The RNA concentration determination, RNA quality control and microarray hybridization were performed according to the manufacturer's instructions from the RNA Labeling kit (Arraystar, Inc., Rockville, MD, USA). Linear RNAs were eliminated using RNase R. The enriched circRNAs were amplified subsequently and transcribed into fluorescent complementary RNA (cRNA). Labeled cRNA was then purified using the RNeasy Mini kit (Qiagen $\mathrm{GmbH}$, Hilden, Germany). Labeled cRNA quality and quantity were determined using the NanoDrop ND-1000 spectrophotometer. Subsequently, the labeled cRNA was fragmented and hybridized onto an Arraystar human circRNA microarray (circular RNA array V2.0 RNA; Arraystar, Inc.). The hybridized arrays were scanned using an Axon GenePix 4000B microarray scanner.

\section{Cell Culture And Proliferation Assay}

As described previously, primary hPASMCs were separated from control subject using distal pulmonary arteries and cultured in high glucose Dulbecco's modified Eagle's medium (DMEM) containing 10\% fetal bovine serum (FBS) at $37^{\circ} \mathrm{C}$. 3-8 passages cells were used for in vitro experiments.

Evaluation of cells proliferation used cell count kit-8 (CCK-8). Cells were seeded into 24-well plates at a

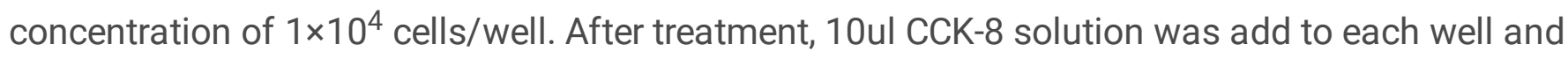
incubated at $37^{\circ} \mathrm{C}$ for $2 \mathrm{~h}$. The absorbance at $450 \mathrm{~nm}$ was determined using ELISA reader.

\section{Qrt-pcr}

Total RNA was isolated with TRIzol Reagent (Invitrogen, Carlsbad, CA, USA). Complementary DNA (cDNA) was synthesized using First-Strand cDNA Synthesis Kit (Invitrogen). The relative mRNA expression were analyzed via RT-PCR using SYBR Green qPCR Mix (TransGen Biotech, Beijing, China). A Roche LightCycler 480 Instrument II was used for real-time quantitative fluorescence analysis with a two-step method. GAPDH and U6 nuclear RNA was used as an internal control. Primer sequences were as follows: 
hsa_circ_0002970: F 5'AGACGAAAGGGGAGGTTATGA3', R 5' TGCAGGTACATTGGTTTCCC3';

hsa_circ_0008199: F 5'AGAATAAAGATAACCAAGACAAGAG3', R 5' TTGCGAAGACACCTGAAGC3';

hsa_circ_0045462 (circ-BPTF): F 5'CCTGTGATGGTACAGCCAGAAA3', R 5'

TGGTGCTGGTTGTACTGCTTGT3';

miR-486-5p: sense GATGCACATCCTGTACTGAGCTG, antisense TATGGTTGTTCACGACTCCTTCAC;

U6: sense CTCGCTTCGGCAGCACA, antisense CTCGCTTCGGCAGCACA

CEMIP: sense CCAGGAATGTTGAATGTCT, anti-sense ATTGGCTCTTGGTGAATG.

GAPDH: F 5'AATCCCATCACCATCTTC3'; R 5'AGGCTGTTGTCATACTTC3'.

\section{Luciferase Assay}

circ-BPTF and CEMIP containing miR-486-5p binding sites were amplified through PCR and cloned into the pmirGLO Dual-Luciferase miRNA Target Expression Vector (Genepharma, Shanghai, PR China). The mutation fragment of the $3^{\prime}$-UTR of CEMIP or circ-BPTF was constructed inserted into the same sites in Dual-Luciferase miRNA Target Expression Vector. Cells were co-transfected with miR-486-5p mimics and pmirGLO vector carrying the desired fragment with Lipofectamine 2000 for $48 \mathrm{~h}$. Luciferase activities were measured by the dual-luciferase reporter assay system (Promega), according to its instructions.

\section{Immunocytochemistry Staining}

After treatment, cells seeding in 24-well plates were fixed with $75 \%$ alcohol for $5 \mathrm{~min}$ and permeabilized with $0.5 \%$ TritonX-100 for $5 \mathrm{~min}$. Cells were incubated with primary antibodies against a-smooth muscle actin or PCNA for $2 \mathrm{~h}$ and subsequent second antibodies for $2 \mathrm{~h}$ at room temperature. Immunoreactivity was visualized by diaminobenzidine (DAB). Negative controls were made with PBS instead of the primary antibodies.

\section{In Situ Detection Of Fragmented Dna (Tunel Assay)}

After treatment, cells seeding in 24-well plates were fixed with $75 \%$ alcohol. The apoptosis in human PASMCs in vitro was evaluated using TUNEL staining kit according to the manufacturer's protocol. TUNEL positive cells percentage were counted and the rate of apoptosis (\%) was calculated as the percentage of TUNEL positive cell nucleus in 5 random fields for each sample.

\section{Western Blot}


Whole protein of cells was extracted and determined using Bradford reagent. Total 50 $\mathrm{\mu g}$ protein was subjected to SDS-PAGE gel. Protein levels was detected by immunobloting with primary antibodies against CEMIP (1:300), cyclin D1 (1:1000). The secondary antibody was horseradish peroxidase (HRP)conjugated goat anti-rabbit lgG. Commercial enhanced chemiluminescence kit was used for immunoreactivity detection. Optical density of visualized blots was analyzed using gel imaging analysis system. The relative density values of each target protein were normalized to GAPDH.

\section{Statistical analysis}

All data were expressed as mean \pm standard deviation and analyzed by $t$ test (between 2 groups) or 2way ANOVA (between more than 2 groups). For all tests, $P<0.05$ was considered statistically significant.

\section{Results}

\section{Clinical data}

All subjects' baseline characteristics are shown in Table 1. There were no differences between the two groups with regard to age and smoking history. As expected from the selected criteria, the forced expiratory volume in 1 second/fixed vital capacity ratio (FEV1/FVC) and forced expiratory volume in 1 second (FEV1\% predicted) in COPD patients was significantly lower than control group (FEV1/FVC: $73.65 \pm 11.59$ vs $94.87 \pm 11.13$, $P<0.01$; FEV1\% predicted: $60.52 \pm 8.75$ vs $79.13 \pm 7.04, P<0.01)$. Although no hypoxemia existed in all patients with operation, the partial pressure of arterial oxygen of COPD patients were markedly lower than that in non-COPD patients $(69.3 \pm 6.2 \mathrm{mmHg}$ vs $82.6 \pm 5.2 \mathrm{mmHg}, P<0.01)$.

Previous report and our recent study have proved the pulmonary vascular remodeling in COPD patients. In this study, we firstly evaluated the pulmonary vascular remodeling in COPD patients. As shown in Figure 1 (A-B), the lung tissue that was HE stained in each patient was focused on intermediate vessels (50$500 \mu \mathrm{m})$ for evaluating pulmonary vascular remodeling. The pulmonary vascular wall thickness and muscularized vessels ratio in COPD patient were both significantly higher than non-COPD patients (Figure 1, C-D).

\section{Circ-bptf Was Upregulated In Lung Of Copd Patietns}

We screened 3610 expressed circRNAs by high-throughput transcriptome sequencing of explants of pulmonary artery from lung tissues of COPD patients or control and 48 differentially expressed circRNAs based on the changes more than 2 folds. Overall, 26 of these circRNAs were significantly upregulated, and 22 were significantly downregulated. We selected 4 significantly upregulated expressed circRNAs and we further verified the expression of the circRNAs by quantitative polymerase chain reaction (qPCR) using the pulmonary arterial smooth muscle strips from lung tissues of COPD patients or controls (Figure 2, AE). Our results showed that circ-BPTF was the most significantly elevated circRNA, consistent with the sequencing results. We then chose circ-BPTF for subsequent analyses. Additionally, we performed 
fluorescence in situ hybridization analysis to determine circ-BPTF distribution and found that circ-BPTF was mainly colocalized with pulmonary arteries (Figure 2F).

Table 1: Baseline characteristic of all patients.

\begin{tabular}{lccc}
\hline & $\begin{array}{c}\text { COPD } \\
(\mathrm{n}=18)\end{array}$ & $\begin{array}{c}\text { non-COPD } \\
(\mathrm{n}=13)\end{array}$ & $p$ value \\
\hline $\mathrm{M} / \mathrm{F}(\mathrm{n})$ & $13 / 5$ & $9 / 4$ & 0.85 \\
\hline Age (yrs) & $64.20 \pm 9.82$ & $57.07 \pm 12.18$ & 0.09 \\
\hline Smoker & 4 & 2 & 0.65 \\
\hline FEV1/pred(\%) & $73.65 \pm 11.59$ & $94.87 \pm 11.13$ & $<0.01$ \\
\hline FEV1/FVC(\%) & $60.52 \pm 8.75$ & $79.13 \pm 7.04$ & $<0.01$ \\
\hline $\mathrm{PaO}_{2}(\mathrm{mmHg})$ & $69.3 \pm 6.2$ & $82.6 \pm 5.2$ & $<0.01$ \\
\hline
\end{tabular}

\section{Circ-bptf Regulated Hpasmcs Proliferation Exposed To Hypoxia}

Next, we investigated the role of circ-BPTF in pulmonary arterial smooth muscle proliferation under hypoxic conditions. Hypoxia induced circ-BPTF upregulation in hPASMCs in vitro as a time-dependent manner (Figure 3A). Specific siRNA targeting circ-BPTF significantly inhibited mRNA levels of circ-BPTF in hPASMCs exposed to hypoxia, and we found that siRNA-1 had the most obvious effects (Figure 3B). Therefore, we transfected hPASMCs with siRNA-1 to study the function of circ-BPTF.

As shown in figure 3C, the results of CCK-8 indicated that hypoxia induced proliferation as a timedependent manner. But siRNA targeting circ-BPTF significantly inhibited proliferation in hypoxia exposing cells. This results were also proved by MTT assay (Figure 3D). We then analyzed the expression of PCNA, a widely used cell proliferation marker. By results from immunocytochemistry staining (Figure 3E and 3F), we showed that hypoxia-induced PCNA over-expression were reversed by knockdown of circ-BPTF following transfection of PASMCs with circ-BPTF siRNA. Additionally, hypoxia decreased the percentage of TUNEL positive cells, which was also reversed after knockdown of circ-BPTF (Figure 3E and 3F).

\section{Circ-bptf Adsorbed Mir-486-5p}


CircRNA acts role as a competitive endogenous sponge for miRNA. We speculated that circ-BPTF might serve as molecular sponge to promote PASMCs proliferation. Based on the bioinformatics software (miRanda and RNAhybrid), we predicted that circ-BPTF might adsorb miR-486-5p, miR-202-3p, miR-758$3 p$ and miR-218-5p (Figure 4A). After exposed to hypoxia, qPCR results showed that miR-486-5p and miR758-3p levels was downregulated, but miR-202-3p and miR-218-5p expression levels was upregulated (Figure 4B). The change of hsa-miR-218-5p was the most significant. Moreover, a recent report has demonstrated the downregulation of miR-486-5p in PASMCs. So, we focused on miR-486-5p in subsequent analyses. We predicted that circ-BPTF and miR-486-5p had 2 binding sites by TargetScan analysis (Figure 4C).

Next, we used a dual luciferase vector plasmid system to verify whether circ-BPTF bound to miR-486-5p. We found that the ratio of luciferase for wild-type circ-BPTF was significantly decreased when compared with that of the negative control after miR-486-5p exposure (Figure 4D). However ratio of luciferase for mutant circ-BPTF did not change as compared with the negative control after miR-486-5p exposure. Moreover, circ-BPTF specific siRNA significantly reversed the suppression of circ-BPTF on miR-486-5p expression (Figure 4E). These results indicated that circ-BPTF could be used as a molecular sponge for miR-486-5p.

\section{Circ-bptf Regulated Cell Proliferation By Adsorbing Mir-486- $5 p$}

To indentify the role of miR-486-5p in cells proliferation, PASMCs transfected with miR-486-5p mimics and exposed to hypoxia for 24-72 hours. The results of CCK-8 showed that miR-486-5p mimics significantly inhibited hypoxia-induced cells proliferation (Figure 5A). The PCNA positive cells were also significantly decreased by miR-486-5p transfection via the results of immunocytochemistry staining (Figure 5B and 5C). Additionally, miR-486-5p mimics increased the TUNEL positive cells percentage (Figure 5B and 5C).

Next we used recovery experiments to identify whether circ-BPTF promoted proliferation via adsorbing miR-486-5p. We co-transfected PASCMCs with circ-BPTF siRNA and miR-486-5p inhibitors before exposure to hypoxia. The results of Edu assays showed that circ-BPTF siRNA or miR-486-5p mimics both inhibited hypoxia-induced proliferation, whereas miR-486-5p inhibitor could reverse the inhibition role of circ-BPTF siRNA on cells proliferation (Figure 5D and 5E). These results demonstrated that circ-BPTF could regulate PASCMCs proliferation by adsorbing miR-486-5p.

\section{Mir-486-5p Targeted Cemip In Pasmcs With Hypoxia Exposure}


Previous reports have demonstrated cell migration-inducing protein (CEMIP) as a down-stream target protein of miR-486-5p, we next try to identify the regulator role of miR-486-5p in CEMIP expression in PASMCs. Based on the bioinformatics software (miRanda and TargetScanHuman ), we predicted two putative binding sites for miR-486-5p on the 3 '-UTR of CEMIP mRNA (Figure 6A). The results of dual luciferase assays showed that ratio of luciferase for wild-type CEMIP was significantly decreased when compared with that of the negative control after miR-486-5p mimics exposure (Figure 6B). However ratio of luciferase for mutant CEMIP did not change as compared with the negative control after miR-486-5p mimics exposure. These results indicated that miR-486-5p could regulate CEMIP expression.

Next, we explored the regulation role of miR-486-5p on CEMIP expression. As shown in Figure 6C, miR486-5p mimics markedly suppressed the mRNA levels of CEMIP in human PASMCs after hypoxia exposure. Furthermore, miR-486-5p mimics significantly inhibited CEMIP protein levels, while miR-486-5p inhibitor raised CEMIP protein levels (Figure 6D and 6E). These results indicated that CEMIP might be a target of miR-486-5p in hypoxia-induced PASMCs proliferation.

\section{Cemip Promoted Proliferation In Pasmcs With Hypoxia Exposure}

Subsequently, we tried to uncover the role of CEMIP in hypoxia-induced PASMCs proliferation. Figure 7A showed the data from GEO database. (GSE56698). Microarray study from lung homogenates revealed that CEMIP is highly up-regulated in hypoxic mice in comparison with those from control mice. Next, we further examined the CEMIP expression in human PASMCs. As shown in Figure 7B and 7C, hypoxia exposure not only raised CEMIP mRNA levels, but also promoted CEMIP protein levels. Moreover, hypoxia raised CEMIP expression as a time-dependent manner (Figure 7C).

Then we used CEMIP specific siRNA to knockdown its expression. In PASMCs exposed to hypoxia, specific siRNA significantly prevented CEMIP expression (Figure 7D). Although hypoxia raised Edu positive cells percentage, CEMIP specific siRNA significantly reversed it as compared with negative control siRNA (Figure 7E and 7F). On the contrary, CEMIP specific siRNA raised TUNEL positive cells in hypoxia-exposed PASMCs (Figure 7G). Additionally, CEMIP specific siRNA prevented the protein expression of p-AKT, cyclin D1 and cIAP2 (Figure 7H and 7I). These results showed that CEMIP might promoted PASMCs proliferation via p-AKT, cyclin D1 and cIAP2 pathways after hypoxia exposure.

\section{Circ-bptf Regulated Mir-486-5p/cemip Axis}

To identify the regulation of circ-BPTF on miR-486-5p/CEMIP Axis, we co-transfected PASCMCs with circBPTF siRNA, miR-486-5p inhibitors and inhibitors before exposure to hypoxia in the recovery experiments. The Western-blot results showed that circ-BPTF specific siRNA and miR-486-5p mimics both could inhibit hypoxia-induced CEMIP protein up-regulation. But miR-486-5p inhibitors reversed the inhibitory effect of circ-BPTF specific siRNA on CEMIP protein expression (Figure 8A and 8B). Circ-BPTF specific siRNA 
suppressed not only the CEMIP expression but also its downstream target, p-AKT (Figure 8C and 8D). These results demonstrated that the role of circ-BPTF/miR-486-5p/CEMIP axis in regulating hypoxiainduced PASMCs proliferation.

\section{Discussion}

In this study, we demonstrated a novel circRNA circ-BPTF and identify the role of circ-BPTF/miR-486$5 p / C E M I P$ axis in regulating hypoxia-induced PASMCs proliferation. Although multiple kinds of circRNAs have been investigated in cancer, only a few reports have focused on circRNA in pulmonary vessels in COPD. This study provided more evidence about circRNA in PASMC proliferation and pulmonary vascular remodeling in COPD.

Although some reports have proved the role of circRNA in cardiovascular disorders and pulmonary hypertension, few studies focused on circRNA in COPD-associated pulmonary vascular remodeling (Yang et al. 2020; Jiang et al. 2021). In this study, we used microarray analysis to identify changes in the expression profiles of circRNAs in lung tissues of COPD patients and in PASMCs exposed to hypoxia. We found a novel circRNA and explored the potential mechanism of circ-BPTF in pulmonary vascular remodeling of COPD by promoting PASMCs proliferation via regulating target miRNA and down-stream protein. As a new class of non-coding RNA molecules, circRNAs are protected from degradation and have greater stability than linear RNA. Numerous circRNAs are expressed in a disease-specific manner. Furthermore, due to other features of circRNAs including stability, conservation and high abundance in body fluids, circRNAs are believed to be potential biomarkers for various diseases. Our this study proved a novel circRNA specific to COPD-associated pulmonary vascular remodeling. It might provided evidence of circRNA as a potential biomarkers.

As a molecular sponge of miRNA, circRNA acts role via securing the target miRNA-mediated suppression of down-stream protein (Boon et al. 2016; Jaé et al. 2020). In this study, we used bioinformatics analysis to predict miRNAs that may bind to circ-BPTF and verified that miR-486-5p could be adsorbed by circBPTF via binding sites at positions 525-532 and 485-504 of circ-BPTF. We further revealed that circ-BPTF promoted PASMCs proliferation by adsorbing miR-486-5p. This result was similar to other reports about circRNA in pulmonary hypertension, which might suggest a circRNA/miRNA pathway underlying PASMCs proliferation and pulmonary vascular remodeling in COPD patients.

Many previous studies have proved that miR-486-5p regulating multiple kinds of cancer cells proliferation (Kong et al. 2020; Hanna et al. 2018; Wang et al. 2014). However, no reports showed the role of miR-486$5 p$ in regulating smooth muscle cells proliferation, especially in PASMCs proliferation. Our reports firstly revealed that miR-486-5p inhibited proliferation in hypoxia-exposed PASMCs. Moreover, we identified the circ-BPTF/miR-486-5p pathway in promoting proliferation in hypoxia condition. MiRNAs are small noncoding RNAs involved in the regulation of posttranscriptional gene expression (Negi et al. 2017). miRNA dysregulations have been reported as promoter of the development of various diseases including cancers (Boateng et al. 2020). Indeed, impairment of miRNA expression has been involved in vascular cell 
remodeling processes such as adventitial fibroblast migration, PASMC proliferation and pulmonary arterial endothelial cell dysfunction observed (Zhou et al. 2015). This study demonstrate the mechanisms through which a novel circRNA (circ-BPTF) regulates hypoxic PASMCs proliferation and pulmonary vascular remodeling in COPD by adsorbing a new miRNA (miR-486-5p). This might enrich the mechanism underlying COPD -associated pulmonary vascular remodeling or pulmonary hypertension.

By inhibiting down-stream target genes, miRNA exert pathophysiological role in multiple physiological and pathological process (Zhou et al. 2018). Bioinformatics analysis in this study showed that CEMIP is a target of miR-486-5p. Although other reports indicated that CEMIP acted critical role in tumor development and proliferation, its role in PASMCs proliferation and vascular remodeling in COPD remain unclear (Deroyer et al. 2019; Rodrigues et al. 2019). This study firstly showed that the CEMIP upregulation and miR-486-5p/CEMIP axis regulating PASMCs proliferation under hypoxia conditions, providing a new potential diagnostic and therapeutic target for this disease. Our previous studies have demonstrated that cyclin D1 participated in pulmonary vascular remodeling in COPD and pulmonary hypertension (Liu et al. 2020; Zeng et al. 2010; Wang et al. 2018). Although some pathways might involved in this process, such as cyclin D1 and p-AKT, it is unclear whether this mechanism participated in pulmonary vascular remodeling in COPD or pulmonary hypertension. In this report, we preliminary showed that cyclin D1, p-AKT and c-IAP2 might be downstream target of CEMIP in PASMCs proliferation. Further studies are still needed to determine the roles of CEMIP and its downstream mechanism in pulmonary vascular remodeling.

In summary, our findings indicated that hypoxia upregulated circ-BPTF. This change resulted in promotion of PASMCs proliferation by adsorption of miR-486-5p, which targeted CEMIP. Thus, we identified a novel circRNA and elucidated the function and mechanisms of this circRNA in PASMCs and pulmonary vascular remodeling in COPD. Future studies are needed to confirm these findings and to provide additional insights into the promising applications of this circRNA.

\section{Abbreviations}

PH: pulmonary hypertension; COPD: Chronic obstructive pulmonary disease; PASMCs: pulmonary arterial smooth muscle cells; CCK-8: cell count kit-8; PCNA: proliferating cell nuclear antigen; PDGF: plateletderived growth factor; DMEM: Dulbecco's modified Eagle's medium; FBS: fetal bovine serum; CEMIP: cell migration-inducing protein.

\section{Declarations}

\section{Ethics approval and consent to participate}

Not applicable.

\section{Consent for publication}


Not applicable.

\section{Availability of data and materials}

The datasets used during the current study are available from the corresponding author on reasonable request.

\section{Competing interests}

All authors declared no potential conflicts of interests.

\section{Funding}

This study was supported by Suzhou Municipal Application Basic Research Project (SYS2019047), Program of Key Talents of Medical Science in Jiangsu Province (QNRC2016745), the Natural Science Foundation of China (No.81970051), Excellent Top Talent Cultivation Project of Anhui Higher Education Institutions (gxgwfx2021014), Applied Medical Research Project of Hefei Health Commission (Hwk2021zd008), scientific research fund from Anhui medical university (2020xkj257).

\section{Authors' contributions}

Wang CG and Liu YY has made substantial contributions to the experiments of the work, the analysis of data and the preparation of manuscript. Zhang WY, Zhang ML, Wang YJ and Huang JA contributed to part of experiment in the study. Zeng DX, Jiang JH and Wang R designed the work, revised the manuscript and approved the submitted version. All authors read and approved the fnal manuscript.

\section{Acknowledgments}

Not applicable.

\section{References}

1. Aufiero S, Reckman YJ, Pinto YM, et al. Circular RNAs open a new chapter in cardiovascular biology. Nat Rev Cardiol. 2019;16:503-514.

2. Blanco I, Piccari L, Barberà JA. Pulmonary vasculature in COPD: The silent component. Respirology. 2016;21:984-994.

3. Boateng E, Krauss-Etschmann S. miRNAs in Lung Development and Diseases. Int J Mol Sci. 2020;21:2765.

4. Bonnet $\mathrm{S}$, Boucherat $\mathrm{O}$, Paulin $\mathrm{R}$, et al. Clinical value of non-coding RNAs in cardiovascular, pulmonary, and muscle diseases. Am J Physiol Cell Physiol. 2020;318:C1-C28.

5. Boon RA, Jaé N, Holdt L, et al. Long Noncoding RNAs: From Clinical Genetics to Therapeutic Targets? J Am Coll Cardiol. 2016;67:1214-1226. 
6. Bunel V, Guyard A, Dauriat G, et al. Pulmonary Arterial Histologic Lesions in Patients With COPD With Severe Pulmonary Hypertension. Chest. 2019;156:33-44.

7. Churg A, Cosio M, Wright JL. Mechanisms of cigarette smoke-induced COPD: insights from animal models. Am J Physiol Lung Cell Mol Physiol. 2008;294:L612-L631.

8. de Jesus Perez VA. Molecular pathogenesis and current pathology of pulmonary hypertension. Heart Fail Rev. 2016;21:239-257.

9. Deroyer C, Charlier E, Neuville S, et al. CEMIP (KIAA1199) induces a fibrosis-like process in osteoarthritic chondrocytes. Cell Death Dis. 2019;10:103.

10. Gredic M, Blanco I, Kovacs G, et al. Pulmonary hypertension in chronic obstructive pulmonary disease. Br J Pharmacol. 2021;178:132-151.

11. Guo HM, Liu ZP. Up-regulation of circRNA_0068481 promotes right ventricular hypertrophy in PAH patients via regulating miR-646/miR-570/miR-885. J Cell Mol Med. 2021;25:3735-3743.

12. Hanna JA, Garcia MR, Lardennois A, et al. PAX3-FOXO1 drives miR-486-5p and represses miR-221 contributing to pathogenesis of alveolar rhabdomyosarcoma. Oncogene. 2018;37:1991-2007.

13. Jaé N, Dimmeler S. Noncoding RNAs in Vascular Diseases. Circ Res. 2020;126:1127-1145.

14. Jiang Y, Liu H, Yu H, et al. Circular RNA Calm4 Regulates Hypoxia-Induced Pulmonary Arterial Smooth Muscle Cells Pyroptosis via the Circ-Calm4/miR-124-3p/PDCD6 Axis. Arterioscler Thromb Vasc Biol. 2021;41:1675-1693.

15. Jin X, Xu Y, Guo M, et al. hsa_circNFXL1_009 modulates apoptosis, proliferation, migration, and potassium channel activation in pulmonary hypertension. Mol Ther Nucleic Acids. 2020;23:10071019.

16. Kong Y, Li Y, Luo Y, et al. circNFIB1 inhibits lymphangiogenesis and lymphatic metastasis via the miR-486-5p/PIK3R1/VEGF-C axis in pancreatic cancer. Mol Cancer. 2020;19:82.

17. Liu YY, Zhang WY, Wang CG, et al. Resveratrol prevented experimental pulmonary vascular remodeling via miR-638 regulating NR4A3/cyclin D1 pathway. Microvasc Res. 2020;130:103988.

18. Ma C, Gu R, Wang X, et al. circRNA CDR1as Promotes Pulmonary Artery Smooth Muscle Cell Calcification by Upregulating CAMK2D and CNN3 via Sponging miR-7-5p. Mol Ther Nucleic Acids. 2020;22:530-541.

19. Negi V, Chan SY. Discerning functional hierarchies of microRNAs in pulmonary hypertension. $\mathrm{JCl}$ Insight. 2017;2:e91327.

20. Nathan SD, Barbera JA, Gaine SP, et al. Pulmonary hypertension in chronic lung disease and hypoxia. Eur Respir J. 2019;53:1801914.

21. Pichl A, Sommer N, Bednorz M, et al. Riociguat for treatment of pulmonary hypertension in COPD: a translational study. Eur Respir J. 2019;53:1802445.

22. Poch D, Mandel J. Pulmonary Hypertension. Ann Intern Med. 2021;174:ITC49-ITC64.

23. Rodrigues G, Hoshino A, Kenific CM, et al. Tumour exosomal CEMIP protein promotes cancer cell colonization in brain metastasis. Nat Cell Biol. 2019;21:1403-1412. 
24. Wang CG, Lei W, Li C, et al. Neuron-derived orphan receptor 1 promoted human pulmonary artery smooth muscle cells proliferation. Exp Lung Res. 2015;41:208-215.

25. Wang CG, Li C, Lei W, et al. The association of neuron-derived orphan receptor 1 with pulmonary vascular remodeling in COPD patients. Int J Chron Obstruct Pulmon Dis. 2018;13:1177-1186.

26. Wang J, Tian X, Han R, et al. Downregulation of miR-486-5p contributes to tumor progression and metastasis by targeting protumorigenic ARHGAP5 in lung cancer. Oncogene. 2014;33:1181-1189.

27. Wang J, Zhu M, Pan J, et al. Circular RNAs: a rising star in respiratory diseases. Respir Res. 2019;20:3.

28. Yang L, Liang H, Meng $X$, et al. mmu_circ_0000790 Is Involved in Pulmonary Vascular Remodeling in Mice with HPH via MicroRNA-374c-Mediated FOXC1. Mol Ther Nucleic Acids. 2020;20:292-307.

29. Zeng DX, Liu XS, Xu YJ, et al. Plasmid-based short hairpin RNA against cyclin D1 attenuated pulmonary vascular remodeling in smoking rats. Microvasc Res. 2010;80:116-122.

30. Zeng DX, Xu GP, Lei W, et al. Suppression of cyclin D1 by plasmid-based short hairpin RNA ameliorated experimental pulmonary vascular remodeling. Microvasc Res. 2013;90:144-149.

31. Zhang J, Li Y, Qi J, et al. Circ-calm4 Serves as an miR-337-3p Sponge to Regulate Myo10 (Myosin 10) and Promote Pulmonary Artery Smooth Muscle Proliferation. Hypertension. 2020;75:668-679.

32. Zhou G, Chen T, Raj JU. MicroRNAs in pulmonary arterial hypertension. Am J Respir Cell Mol Biol. 2015;52:139-151.

33. Zhou S, Jiang H, Li M, et al, Wang R. Circular RNA hsa_circ_0016070 Is Associated with Pulmonary Arterial Hypertension by Promoting PASMC Proliferation. Mol Ther Nucleic Acids. 2019;18:275-284.

34. Zhou SS, Jin JP, Wang JQ, et al. miRNAS in cardiovascular diseases: potential biomarkers, therapeutic targets and challenges. Acta Pharmacol Sin. 2018;39:1073-1084.

\section{Figures}




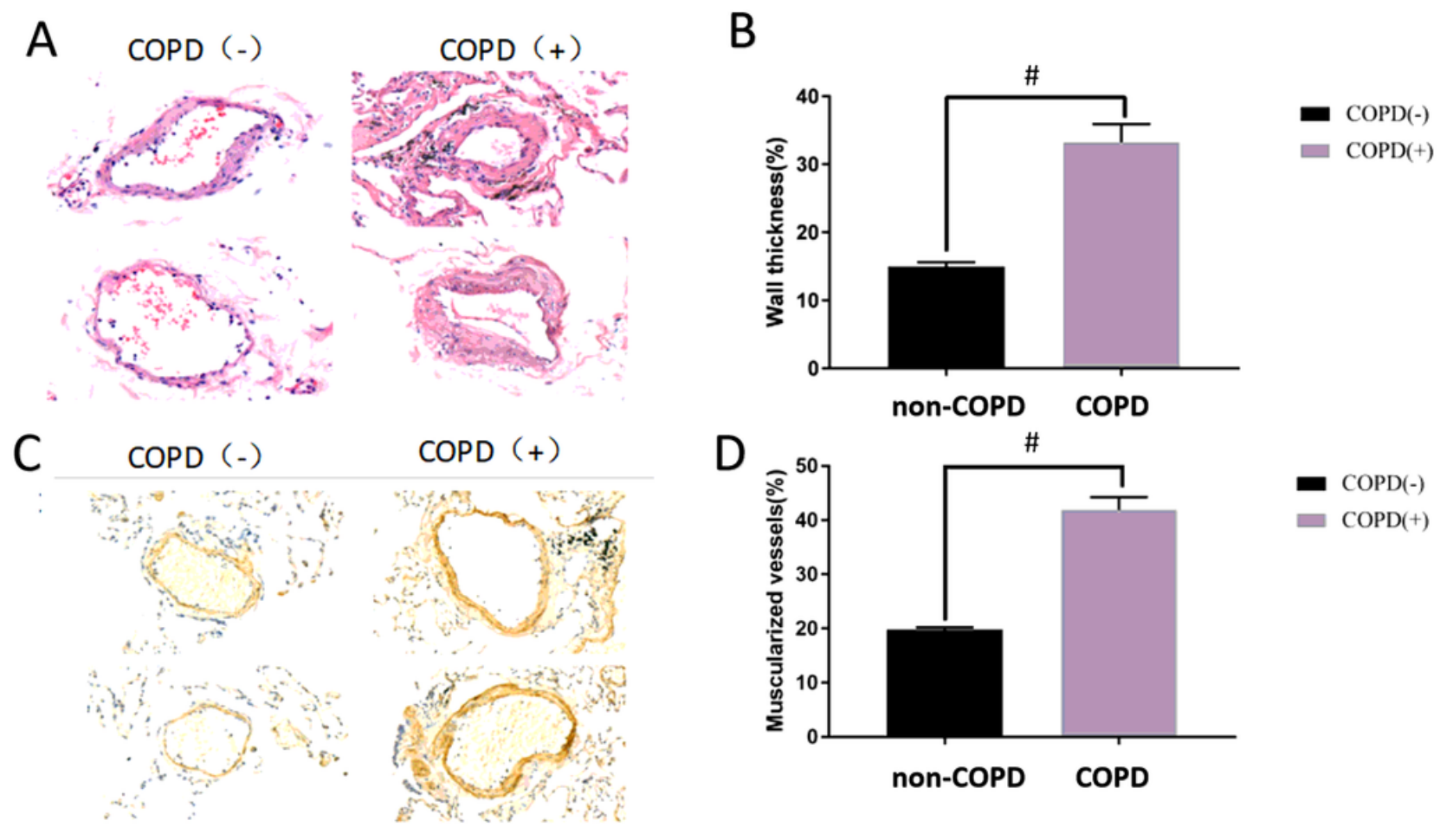

Figure 1

Pulmonary vascular remodeling in COPD patients. Lung tissues were collected in COPD or non-COPD patients and pulmonary vascular remodeling was estimated. (A-B) HE staining showed the wall thickness of pulmonary vessels in patients. (C-D) Immunohistochemistry staining (a-SMA) showed the muscularized vessels of pulmonary vessels in patients. $\otimes \mathrm{P}<0.05$. 
A
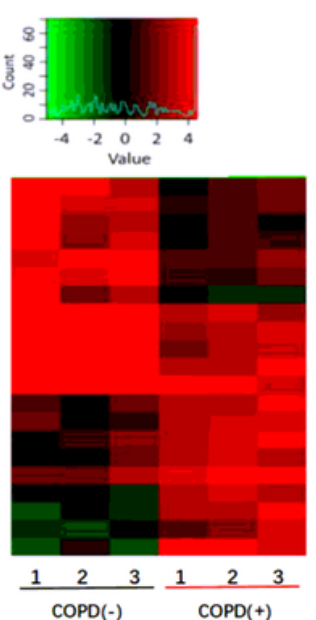

hsa_circ_0081386 hisa circ 0056297 hasa circ 0007334 lisa_cire 0004417 hsa_circ_0008323 lisa_cire_0000973 tisa- 0069714 is hisa_cir 0007440 hisa_circ_008413 hisa circ 000947 lasa_circ_000714 thsa_dir_ _0060s4 bas iss. bascir 000106 iss circ 0045462 (circ-BPTF) $\mathrm{COPD}(-) \quad \mathrm{COPD}(+)$
B

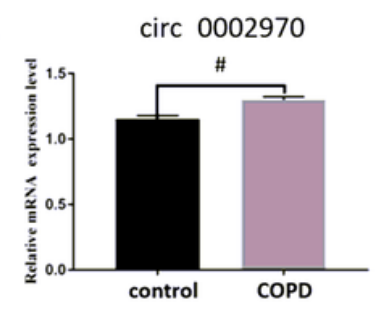

D

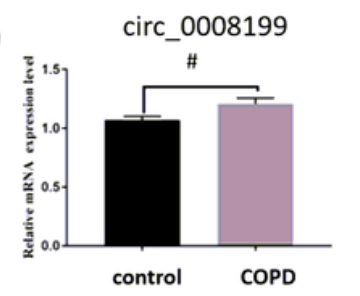

C circ_0004893

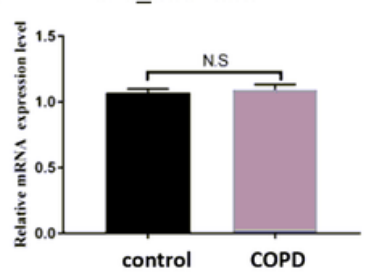

E

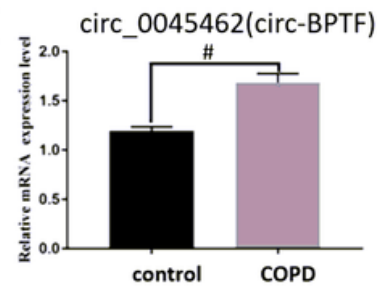

F

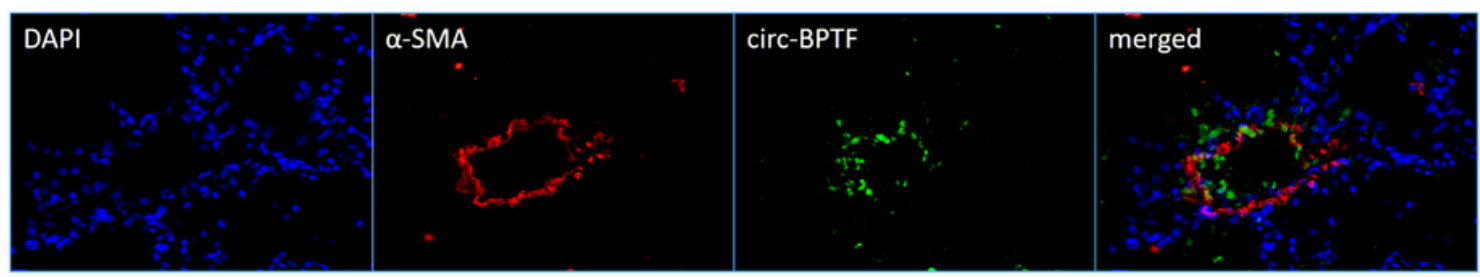

Figure 2

Circ-BPTF was upregulated in lung and smooth muscle of COPD patients. Lung tissues were collected form COPD or control patients. Total RNA was isolated and and circRNA microarray hybridization were performed based on Arraystar's standard protocols. (A) Heat map and volcano map analysis of circular RNAs in lung tissues from COPD patients or controls. (B-E) qPCR analysis of four circRNAs expression in lung from COPD and controls $(n=6)$. ( $F$ ) Fluorescence in situ hybridization of circ-BPTF in lung tissues from COPD patients. $\otimes P<0.05$ 

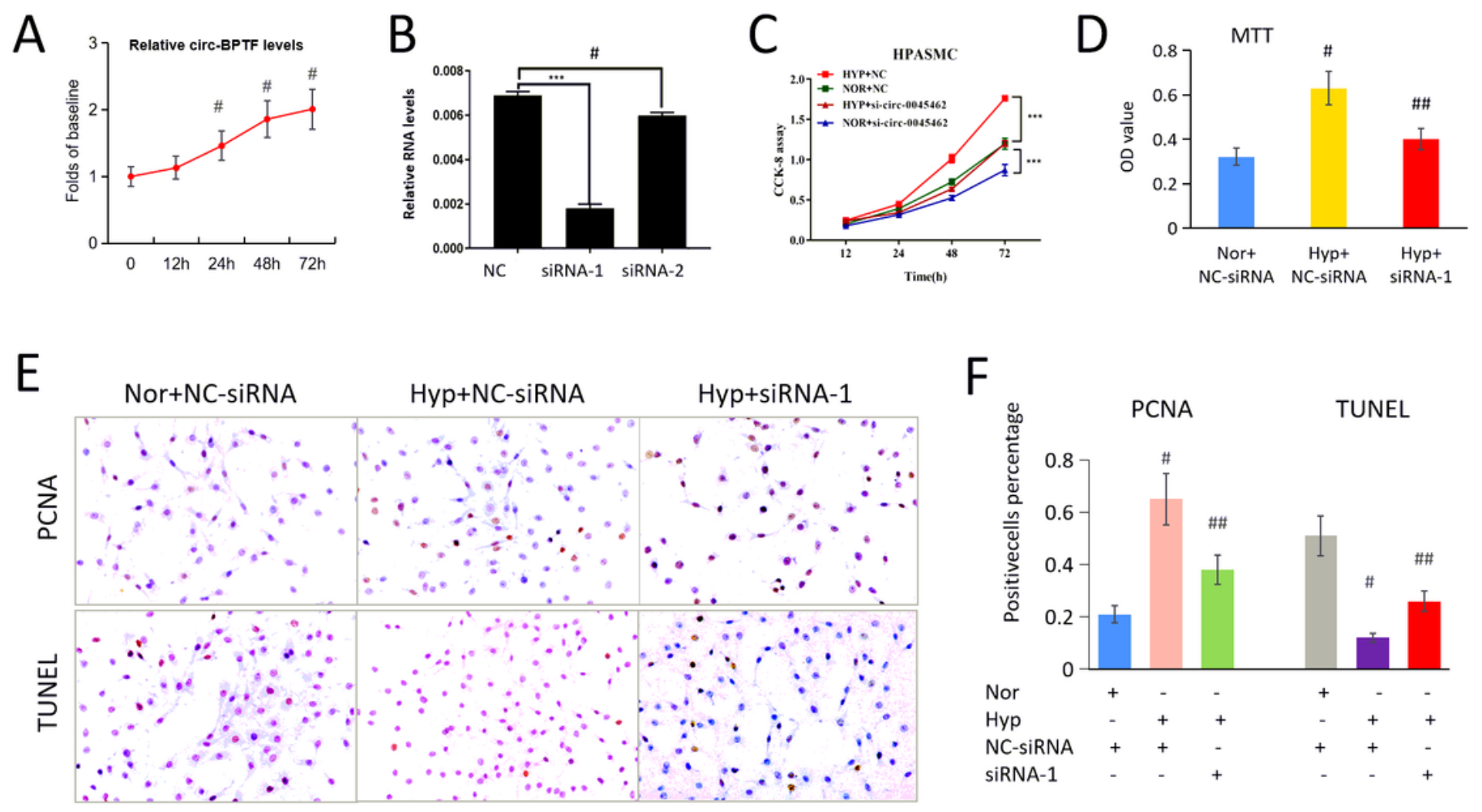

\section{Figure 3}

Circ-BPTF regulated PASMCs proliferation exposed to hypoxia. Human PASMCs were cultured in vitro under hypoxia or normoxia. Specific siRNA was used to knockdown circ-BPTF expression. (A) qPCR analysis of circ-BPTF levels after exposure to hypoxia for different time . (B) Specific siRNA knockdown circ-BPTF expression in hypoxia-exposed PASMCs. (C-D) CCK-8 and MTT assay showed the circ-BPTF specific siRNA suppressed cells proliferation in hypoxia-exposed PASMCs. (E-F) Immunohistochemistry staining and the TUNEL assay showed the circ-BPTF specific siRNA suppressed PCNA-positive cells and

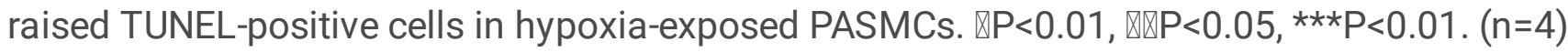



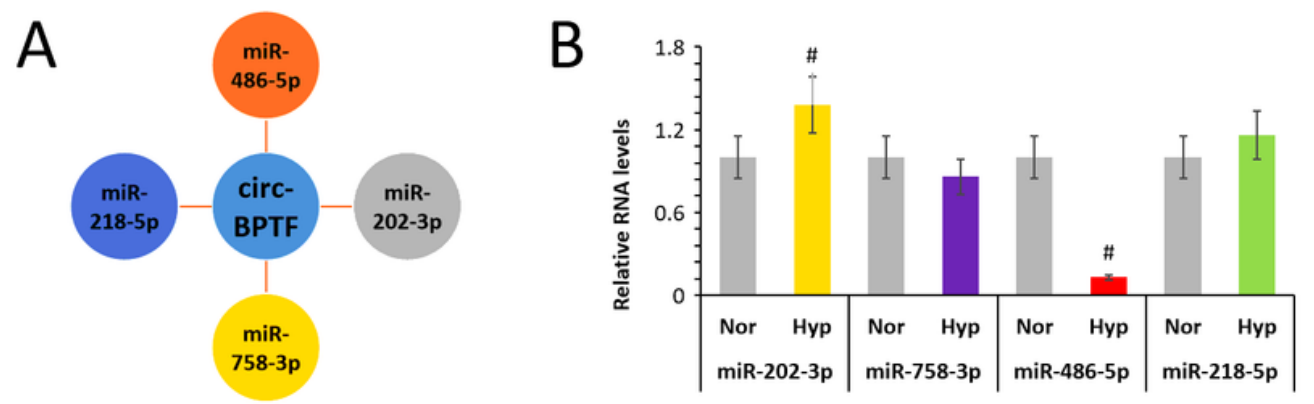
C circ-BPTF, chr 17: 65941525-65941532: 5' circ-BPTF, chr 17: 65925482-65925504: 5'guaucauucca UCAA GUACAGG u 3' miR-486-5p: GAGCCCCGUCGAGU CAUGUCC U
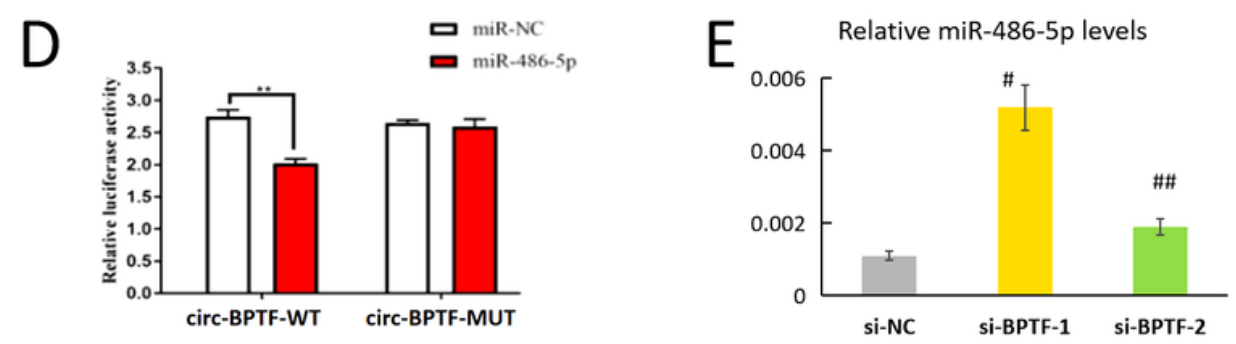

\section{Figure 4}

Circ-BPTF adsorbed miR-486-5p. (A) Bioinformatics software (miRanda and RNAhybrid) predicted miRNAs that could bind to circ-BPTF. (B) Human PASMCs were exposed to hypoxia for $24 \mathrm{~h}$ and qPCR was used to detect miR-202-3p, miR-758-3p, miR-486-5p and miR-218-5p expression levels . (C) The predicted binding sites of circ-BPTF and miR-486-5p by TargetScan. (D) Dual luciferase assays demonstrated the interactions between circ-BPTF and miR-486-5p. (E) Human PASMCs were transfected with circ-BPTF specific siRNA (si-BPTF) or negative control siRNA (si-NC) before exposing to hypoxia for 24h. qPCR was used for detection of miR-486-5p levels. $\triangle P<0.01$, $\otimes \mathbb{Q}<<0.05, \star \star P<0.01 .(n=4)$ 
A

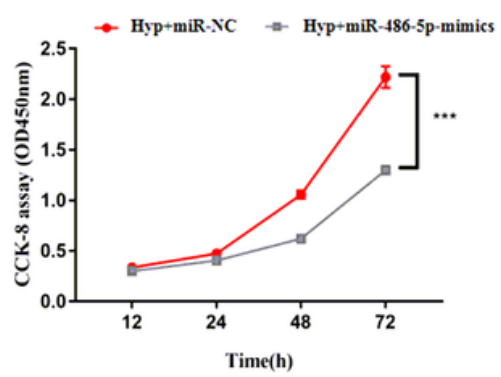

B

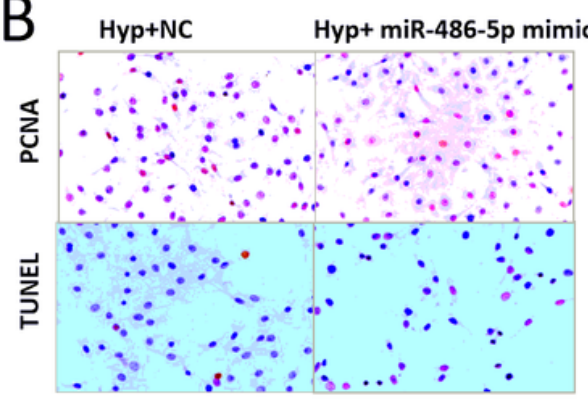

D

Hoechst33342

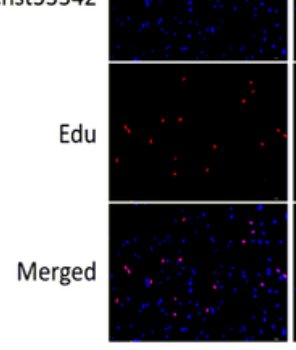

Nor

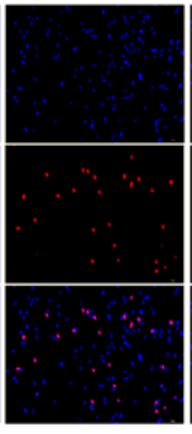

Nor+ inhibitor

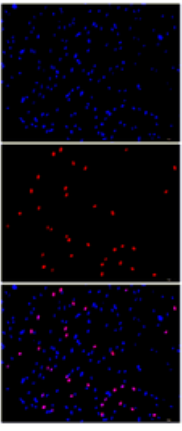

Hyp

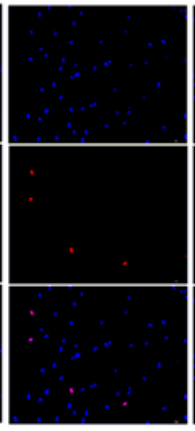

Hyp+ mimics

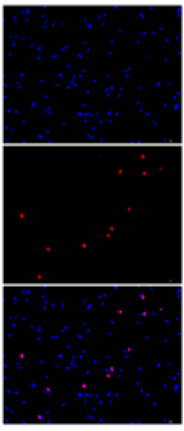

Hyp+ si-circ

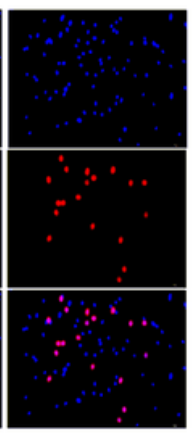

Hyp+ si-circ + inhibitor
C
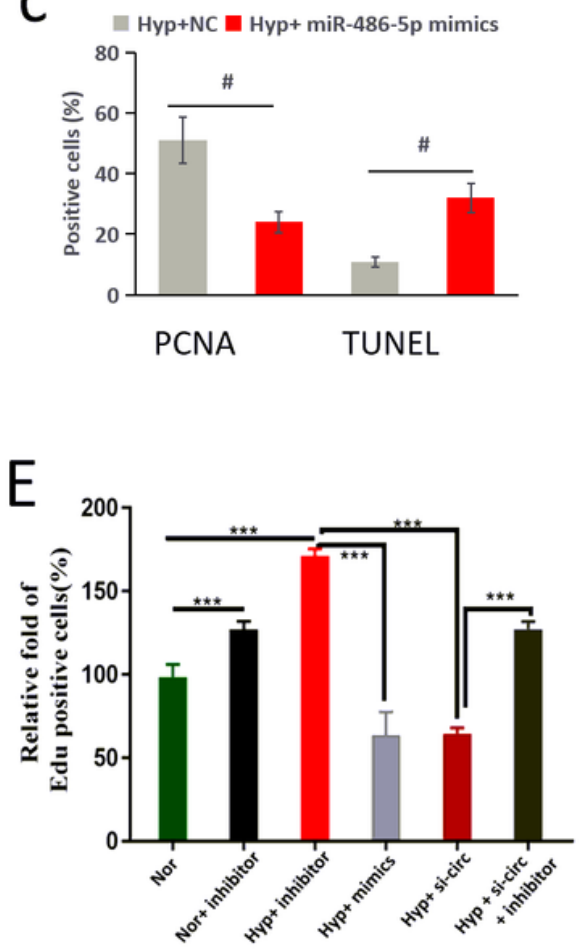

\section{Figure 5}

Circ-BPTF regulated cell proliferation by adsorbing miR-486-5p. Human PASMCs were cultured in vitro under hypoxia or normoxia. Cells were transfected with miR-486-5p mimics, miR-486-5p inhibitor or circBPTF specific siRNA. (A) CCK-8 assay showed that miR-486-5p mimics inhibited proliferation in hypoxia cells. (B-C) Immunohistochemistry staining and the TUNEL assay showed that miR-486-5p mimics suppressed PCNA-positive cells percentage but raised TUNEL-positive cells percentage. (D-E) Immunofluorescence staining for Edu assay showed that circ-BPTF siRNA or miR-486-5p mimics both inhibited hypoxia-induced Edu-positive cells percentage, whereas miR-486-5p inhibitor could reverse this inhibition effect. (Inhibitor: miR-486-5p inhibitor; mimics: miR-486-5p mimics; sic-circ: circ-BPTF specific siRNA) $\otimes P<0.01, \star \star \star P<0.05 .(n=4)$ 
A

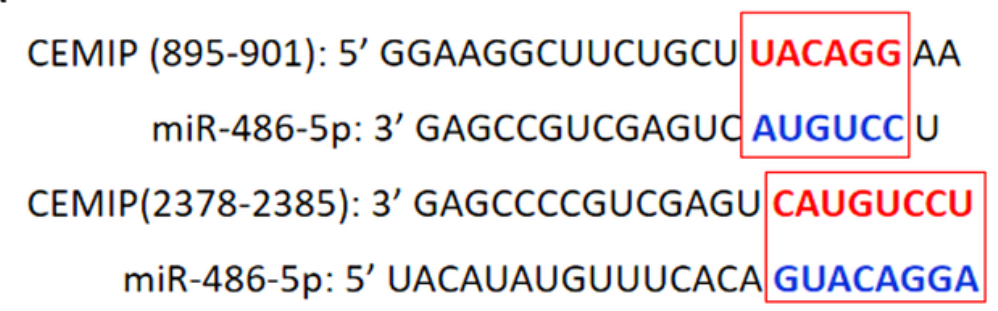

B

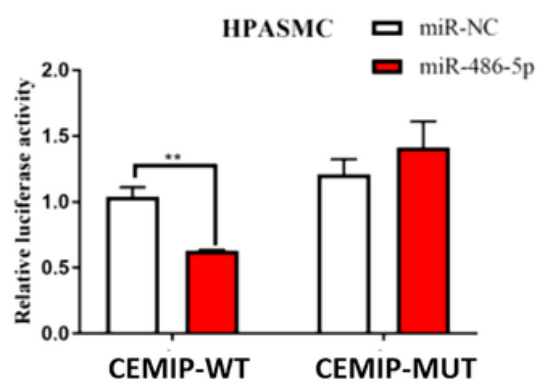

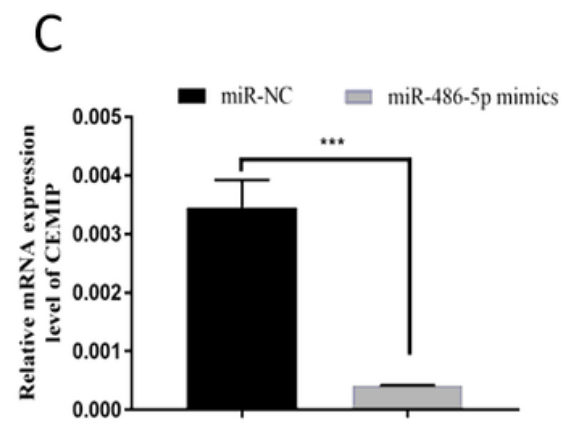

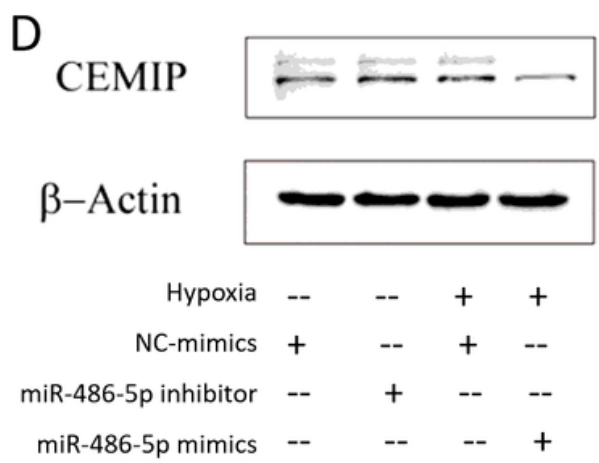

$E$

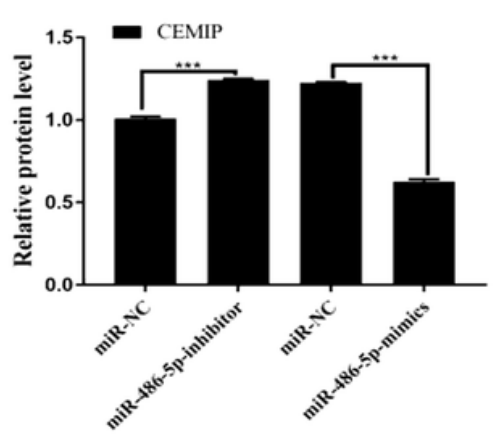

Figure 6

miR-486-5p targeted CEMIP. (A) TargetScan predicted the binding sites of miR-486-5p and CEMIP. (B) Dual luciferase assays were used to identify the interactions between miR-486-5p and CEMIP. (C) qPCR showed that miR-486-5p mimics inhibited CEMIP expression in hypoxic PASMCs. (D-E) Western-blot results verified that miR-486-5p inhibitor upregulated CEMIP protein levels but miR-486-5p mimics downregulated CEMIP protein levels. ${ }^{\star *} P<0.05,{ }^{\star} * \star P<0.01$. $(n=4)$ 
A

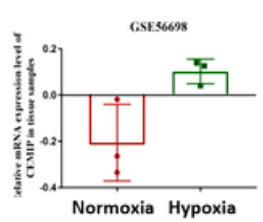

B

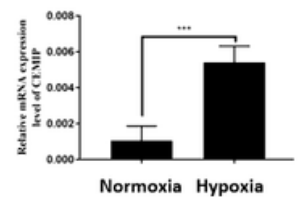

$\mathrm{F}$

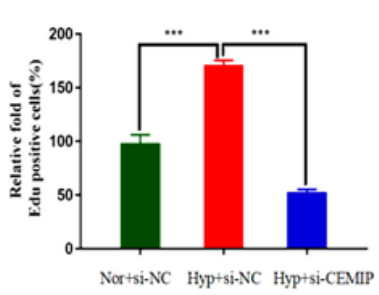

C

G
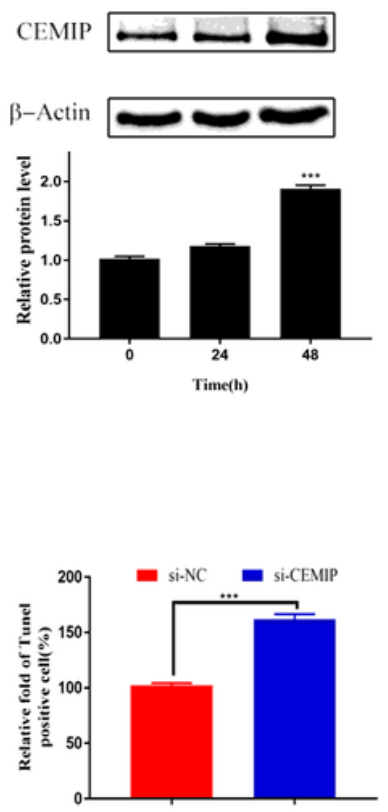

D

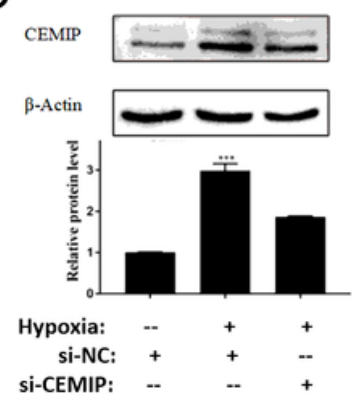

$\mathrm{H}$

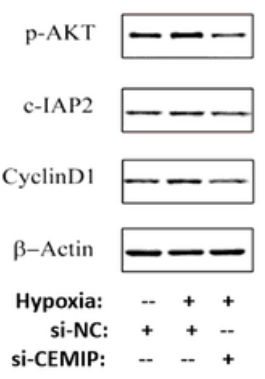

$\mathrm{E}$

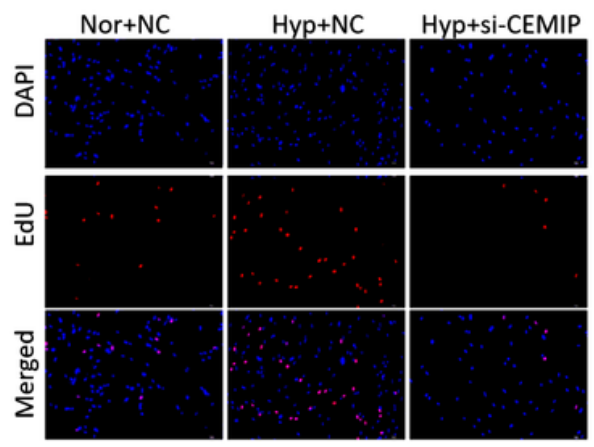

\section{Figure 7}

CEMIP promoted proliferation in PASMCs with hypoxia exposure. Human PASMCs were cultured in vitro under hypoxia or normoxia. Cells were transfected with CEMIP specific siRNA or negative control siRNA. (A) Microarray study about lung homogenates from GEO database (GSE56698) showed the upregulation of CEMIP in hypoxic mice. (B) qPCR detected the CEMIP mRNA levels in hypoxic PASMCS. (C) Westernblot results showed the upregulation of CEMIP protein levels in hypoxic PASMCs with a time-dependent manner. (D) Specific siRNA (si-CEMIP) suppressed CEMIP protein levels in hypoxic PASMCs. (E-F) Immunofluorescence staining for Edu assay showed that si-CEMIP inhibited Edu-positive cells percentage in hypoxic PASMCs. (G) TUNNEL assay showed that si-CEMIP raised TUNNEL-positive cells percentage in

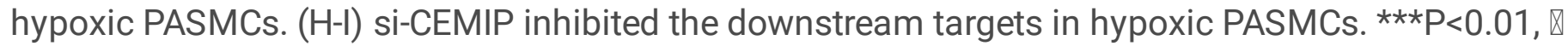
$P<0.01$, $\mathbb{Q} \mathbb{P}<0.05 .(n=4)$ 

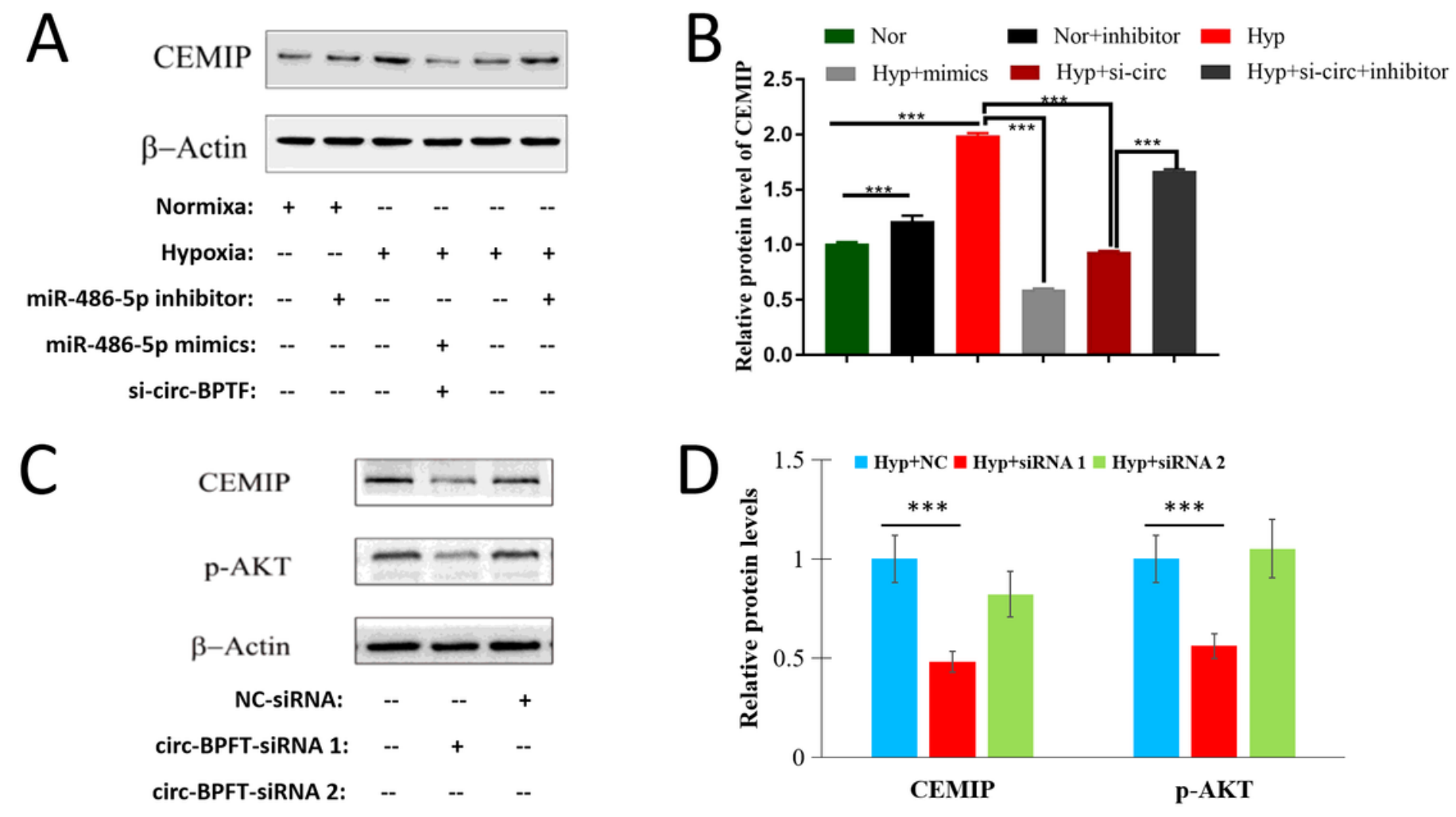

\section{Figure 8}

Circ-BPTF regulated miR-486-5p/CEMIP Axis. Human PASMCs were cultured in vitro under hypoxia or normoxia. Cells were co-transfected with miR-486-5p mimics, miR-486-5p inhibitor or circ-BPTF specific siRNA. (A-B) Western-blot results showed the effect of miR-486-5p mimics, miR-486-5p inhibitor and circBPTF specific siRNA on CEMIP protein levels under hypoxia or normoxia. Circ-BPTF specific siRNA and miR-486-5p mimics could both inhibit CEMIP protein levels in hypoxic PASMCs, but miR-486-5p inhibitors reversed this inhibitory effect. (C-D) Western-blot results showed the inhibitory effect of circ-BPTF specific siRNA on CEMIP protein levels and its downstream targets in in hypoxic PASMCs. ${ }^{\star \star *} P<0.01 .(n=4)$ 